\title{
Amplitudes for massive vector and scalar bosons in spontaneously-broken gauge theory from the $\mathrm{CHY}$ representation
}

\section{Stephen G. Naculich}

Department of Physics, Bowdoin College, Brunswick, ME 04011, U.S.A.

E-mail: naculich@bowdoin.edu

ABSTRACT: In the formulation of Cachazo, He, and Yuan, tree-level amplitudes for massless particles in gauge theory and gravity can be expressed as rational functions of the Lorentz invariants $k_{a} \cdot k_{b}, \epsilon_{a} \cdot k_{b}$, and $\epsilon_{a} \cdot \epsilon_{b}$, valid in any number of spacetime dimensions. We use dimensional reduction of higher-dimensional amplitudes of particles with internal momentum $\kappa$ to obtain amplitudes for massive particles in lower dimensions. In the case of gauge theory, we argue that these massive amplitudes belong to a theory in which the gauge symmetry is spontaneously broken by an adjoint Higgs field. Consequently, we show that tree-level $n$-point amplitudes containing massive vector and scalar bosons in this theory can be obtained by simply replacing $k_{a} \cdot k_{b}$ with $k_{a} \cdot k_{b}-\kappa_{a} \kappa_{b}$ in the corresponding massless amplitudes, where the masses of the particles are given by $\left|\kappa_{a}\right|$.

Keywords: Spontaneous Symmetry Breaking, Scattering Amplitudes, Duality in Gauge Field Theories, Field Theories in Higher Dimensions

ARXIV EPRINT: 1506.06134 
In a series of papers over the last few years, Cachazo, He, and Yuan (CHY) have developed a new representation for the tree-level amplitudes of massless particles in a variety of theories, including Yang-Mills theory and Einstein gravity [1-3], as well as the nonlinear $\sigma$ model, the Dirac-Born-Infeld theory, and more exotic models [4]. In this approach, the ingredients for an $n$-point amplitude are the positions $\sigma_{a}$ of $n$ punctures on a sphere, as well as Lorentz invariants of the momenta $k_{a}^{\mu}$ and polarizations $\epsilon_{a}^{\mu}$ of the particles. (The graviton polarization is expressed as $\epsilon_{a}^{\mu \nu}=\epsilon_{a}^{\mu} \tilde{\epsilon}_{a}^{\nu}$.) The CHY representation involves an integral over the moduli space of the punctured sphere, which can be evaluated $[2,4-6]$ to yield a rational expression of $k_{a} \cdot k_{b}, \epsilon_{a} \cdot k_{b}$, and $\epsilon_{a} \cdot \epsilon_{b}$ for the $n$-point tree-level amplitude.

A notable feature of this formulation is its independence of the number of spacetime dimensions. One can therefore dimensionally reduce CHY representations of gravity or Yang-Mills amplitudes in $(d+M)$ dimensions to obtain mixed amplitudes in $d$ dimensions containing gravitons, gauge bosons, and massless scalars by choosing the $(d+M)$ dimensional polarizations $\mathcal{E}_{a}$ to lie in either the $d$ - or $M$-dimensional subspace $[3,4]$.

Dimensional reduction can also be used to endow particles with mass, and therefore to obtain CHY representations for massive particles in $d$ dimensions [7]. (Previous extensions of the CHY approach to massive particles were considered in refs. [8-10].) The momentum of a particle in $(d+M)$ dimensions can be written

$$
K_{a}=\left(k_{a} \mid \kappa_{a}\right)
$$

where $k_{a}$ and $\kappa_{a}$ are the components of momentum in $d$ - and $M$-dimensional subspaces respectively; we will refer to $\kappa_{a}$ as the internal momentum of a particle, and regard it as a fixed quantity. A massless particle in $(d+M)$ dimensions with momentum $K_{a}$ corresponds to a particle in $d$ dimensions with mass $m_{a}=\left|\kappa_{a}\right|$. This approach is similar to that used in refs. [11-13], where a fifth dimension was introduced as a massive infrared regulator for planar loop-level $\mathcal{N}=4$ amplitudes. See also, for example, refs. [14-18], in which massive particles are represented as higher-dimensional massless particles.

The amplitude for massless gauge bosons in $(d+M)$ dimensions, which is a rational function of $K_{a} \cdot K_{b}, \mathcal{E}_{a} \cdot K_{b}$, and $\mathcal{E}_{a} \cdot \mathcal{E}_{b}$, can be used to obtain an expression for the amplitude for massive gauge bosons in $d$ dimensions by choosing $\mathcal{E}_{a}=\left(\epsilon_{a} \mid 0\right)$, so that ${ }^{1}$

$$
K_{a} \cdot K_{b}=k_{a} \cdot k_{b}-\kappa_{a} \cdot \kappa_{b}, \quad \mathcal{E}_{a} \cdot K_{b}=\epsilon_{a} \cdot k_{b}, \quad \mathcal{E}_{a} \cdot \mathcal{E}_{b}=\epsilon_{a} \cdot \epsilon_{b} .
$$

In other words, we simply replace $k_{a} \cdot k_{b}$ with $k_{a} \cdot k_{b}-\kappa_{a} \cdot \kappa_{b}$ in the $d$-dimensional massless gauge boson amplitude. The question remains: to what theory do these massive amplitudes belong?

Not all $d$-dimensional amplitudes of massive particles can be obtained from dimensional reduction of $(d+M)$-dimensional massless amplitudes, due to the constraints arising from internal momentum conservation. Overall momentum conservation demands $\sum_{a=1}^{n} \kappa_{a}=$ 0 , placing a restriction on the masses of a dimensionally-reduced $n$-particle amplitude. Furthermore, internal momentum conservation must be satisfied at each vertex of the

\footnotetext{
${ }^{1}$ The relative minus sign in $K_{a} \cdot K_{b}$ arises because we use a mostly-minus metric for $K_{a}$ and $k_{a}$, but an all-plus metric for the internal components $\kappa_{a}$.
} 
tree diagrams that contribute to the amplitude, constraining the mass of any particle propagating in intermediate channels. For example, if particles $a$ and $b$ couple into an intermediate channel, the mass of the particle in that channel must be given by $\left|\kappa_{a}+\kappa_{b}\right|$. In ref. [7], it was shown that this constraint is automatically satisfied in an amplitude with no more than three massive particles, in which the remaining massless particles are flavor-preserving, and therefore such amplitudes can be given a CHY representation. But for more general massive amplitudes (e.g. in the standard model), the internal momentum constraints cannot be satisfied.

It is the purpose of this note to observe that the internal momentum constraints can be satisfied in a gauge theory in which the $\mathrm{U}(N)$ symmetry is spontaneously broken by an adjoint Higgs field $H$. If the Higgs field has vacuum expectation value $\langle H\rangle=$ $\operatorname{diag}\left(v_{1}, v_{2}, \cdots, v_{N}\right)$, the $\mathrm{U}(N)$ symmetry is broken to $\mathrm{U}(1)^{N}$, and the off-diagonal gauge bosons $W_{i}^{j}$ obtain masses $g\left|v_{i}-v_{j}\right|$ from the $\operatorname{Tr}\left(D_{\mu} H\right)^{2}$ term in the Lagrangian. If some of the $v_{i}$ are degenerate, then a larger symmetry will be left unbroken. (This is essentially the theory considered in refs. [11,15,18-23], where it is embedded in an $\mathcal{N}=4$ supersymmetric theory on the Coulomb branch. In the string theory picture, the $v_{i}$ correspond to the positions of D3 branes in the fifth dimension, and massive gauge bosons correspond to strings extending between separated D3 branes. See also refs. [24, 25] for tree-level massive amplitudes on the Coulomb branch of this theory.)

At a triple-gauge-boson vertex, $W_{i}{ }^{j}$ and $W_{j}{ }^{k}$ couple to $W_{i}{ }^{k}$. If we identify the internal momentum ${ }^{2} \kappa_{i j}$ of $W_{i}^{j}$ with $g\left(v_{i}-v_{j}\right)$, then internal momentum conservation $\kappa_{i j}+\kappa_{j k}=$ $\kappa_{i k}$ automatically holds at each vertex (and similarly at four-gauge-boson vertices). This suggests that dimensionally-reduced amplitudes with internal momenta $\kappa$ are equivalent to amplitudes of massive gauge bosons in this spontaneously-broken theory. Moreover, this implies that massive amplitudes in this theory can be obtained from the corresponding massless amplitudes of the unbroken theory, expressed in terms of $k_{a} \cdot k_{b}, \epsilon_{a} \cdot k_{b}$, and $\epsilon_{a} \cdot \epsilon_{b}$, by simply replacing $k_{a} \cdot k_{b}$ with $k_{a} \cdot k_{b}-\kappa_{a} \kappa_{b}$. To illustrate this in a simple case, we evaluate the amplitude of four massive gauge bosons in the spontaneously-broken theory

$$
\begin{aligned}
\left\langle W_{i_{1}}^{i_{2}}\left(k_{1}, \epsilon_{1}\right) W_{i_{2}}^{i_{3}}\left(k_{2}, \epsilon_{2}\right) W_{i_{3}}^{i_{4}}\left(k_{3}, \epsilon_{3}\right) W_{i_{4}}^{i_{1}}\left(k_{4}, \epsilon_{4}\right)\right\rangle & \\
= & g^{2}\left[\frac{N_{s}}{\left(k_{1}+k_{2}\right)^{2}-m_{s}^{2}}+\frac{N_{t}}{\left(k_{1}+k_{4}\right)^{2}-m_{t}^{2}}+(\text { contact term })\right]
\end{aligned}
$$

which have contributions from $s$ - and $t$-channel exchange as well as a four-gauge-boson contact term. For brevity, we include below only those terms in the numerators that depend on $k_{a} \cdot k_{b}$; the omitted terms are the same as those in the unbroken theory:

$$
\begin{aligned}
& N_{s}=\frac{\left(\epsilon_{1} \cdot \epsilon_{2}\right)\left(\epsilon_{3} \cdot \epsilon_{4}\right)}{2}\left[\left(k_{2}-k_{1}\right) \cdot\left(k_{4}-k_{3}\right)+\frac{\left(m_{2}^{2}-m_{1}^{2}\right)\left(m_{4}^{2}-m_{3}^{2}\right)}{m_{s}^{2}}\right]+\cdots, \\
& N_{t}=\frac{\left(\epsilon_{1} \cdot \epsilon_{4}\right)\left(\epsilon_{2} \cdot \epsilon_{3}\right)}{2}\left[\left(k_{2}-k_{3}\right) \cdot\left(k_{4}-k_{1}\right)+\frac{\left(m_{1}^{2}-m_{4}^{2}\right)\left(m_{3}^{2}-m_{2}^{2}\right)}{m_{t}^{2}}\right]+\cdots .
\end{aligned}
$$

\footnotetext{
${ }^{2}$ now restricted to one dimension
} 
Here $m_{a}=\left|\kappa_{a}\right|$ with $\kappa_{a} \equiv g\left(v_{i_{a}}-v_{i_{a+1}}\right)$, where $i_{5} \equiv i_{1}$ so that $\sum_{a=1}^{4} \kappa_{a}=0 . \quad$ Up to this point, we have not made any assumptions about the masses of the $s$ and $t$-channel intermediate particles. Since the particles propagating in those channels are $W_{i_{1}}{ }^{i_{3}}$, and $W_{i_{2}}^{i_{4}}$ respectively, we have

$$
m_{s}^{2}=g^{2}\left(v_{i_{1}}-v_{i_{3}}\right)^{2}=\left(\kappa_{1}+\kappa_{2}\right)^{2}, \quad m_{t}^{2}=g^{2}\left(v_{i_{2}}-v_{i_{4}}\right)^{2}=\left(\kappa_{2}+\kappa_{3}\right)^{2}
$$

and the numerators above simplify to

$$
\begin{aligned}
& N_{s}=\frac{1}{2}\left(\epsilon_{1} \cdot \epsilon_{2}\right)\left(\epsilon_{3} \cdot \epsilon_{4}\right)\left[\left(k_{2}-k_{1}\right) \cdot\left(k_{4}-k_{3}\right)-\left(\kappa_{2}-\kappa_{1}\right)\left(\kappa_{4}-\kappa_{3}\right)\right]+\cdots \\
& N_{t}=\frac{1}{2}\left(\epsilon_{1} \cdot \epsilon_{4}\right)\left(\epsilon_{2} \cdot \epsilon_{3}\right)\left[\left(k_{2}-k_{3}\right) \cdot\left(k_{4}-k_{1}\right)-\left(\kappa_{2}-\kappa_{3}\right)\left(\kappa_{4}-\kappa_{1}\right)\right]+\cdots
\end{aligned}
$$

We assemble all the pieces and use

$$
\begin{aligned}
& \left(k_{1}+k_{2}\right)^{2}-m_{s}^{2}=2\left(k_{1} \cdot k_{2}-\kappa_{1} \kappa_{2}\right)=2\left(k_{3} \cdot k_{4}-\kappa_{3} \kappa_{4}\right), \\
& \left(k_{1}+k_{4}\right)^{2}-m_{t}^{2}=2\left(k_{1} \cdot k_{4}-\kappa_{1} \kappa_{4}\right)=2\left(k_{2} \cdot k_{3}-\kappa_{2} \kappa_{3}\right)
\end{aligned}
$$

to obtain

$$
\left\langle W_{i_{1}}^{i_{2}}\left(k_{1}, \epsilon_{1}\right) W_{i_{2}}^{i_{3}}\left(k_{2}, \epsilon_{2}\right) W_{i_{3}}^{i_{4}}\left(k_{3}, \epsilon_{3}\right) W_{i_{4}}^{i_{1}}\left(k_{4}, \epsilon_{4}\right)\right\rangle=-g^{2} \frac{\mathbf{K}}{\left(k_{2} \cdot k_{3}-\kappa_{2} \kappa_{3}\right)\left(k_{3} \cdot k_{4}-\kappa_{3} \kappa_{4}\right)}
$$

with

$$
\begin{aligned}
\mathbf{K}=-\{ & \left(k_{1} \cdot k_{3}-\kappa_{1} \kappa_{3}\right)\left(k_{2} \cdot k_{3}-\kappa_{2} \kappa_{3}\right) \epsilon_{1} \cdot \epsilon_{2} \epsilon_{3} \cdot \epsilon_{4} \\
& +\left(k_{2} \cdot k_{3}-\kappa_{2} \kappa_{3}\right)\left(k_{3} \cdot k_{4}-\kappa_{3} \kappa_{4}\right) \epsilon_{1} \cdot \epsilon_{3} \epsilon_{2} \cdot \epsilon_{4} \\
& +\left(k_{1} \cdot k_{3}-\kappa_{1} \kappa_{3}\right)\left(k_{3} \cdot k_{4}-\kappa_{3} \kappa_{4}\right) \epsilon_{1} \cdot \epsilon_{4} \epsilon_{2} \cdot \epsilon_{3} \\
& +\left[\left(k_{1} \cdot k_{3}-\kappa_{1} \kappa_{3}\right) \epsilon_{1} \cdot k_{4} \epsilon_{2} \cdot k_{3}+\left(k_{2} \cdot k_{3}-\kappa_{2} \kappa_{3}\right) \epsilon_{1} \cdot k_{3} \epsilon_{2} \cdot k_{4}\right] \epsilon_{3} \cdot \epsilon_{4} \\
& +\left[\left(k_{2} \cdot k_{3}-\kappa_{2} \kappa_{3}\right) \epsilon_{1} \cdot k_{2} \epsilon_{3} \cdot k_{4}+\left(k_{3} \cdot k_{4}-\kappa_{3} \kappa_{4}\right) \epsilon_{1} \cdot k_{4} \epsilon_{3} \cdot k_{2}\right] \epsilon_{2} \cdot \epsilon_{4} \\
& +\left[\left(k_{1} \cdot k_{3}-\kappa_{1} \kappa_{3}\right) \epsilon_{1} \cdot k_{2} \epsilon_{4} \cdot k_{3}+\left(k_{3} \cdot k_{4}-\kappa_{3} \kappa_{4}\right) \epsilon_{1} \cdot k_{3} \epsilon_{4} \cdot k_{2}\right] \epsilon_{2} \cdot \epsilon_{3} \\
& +\left[\left(k_{1} \cdot k_{3}-\kappa_{1} \kappa_{3}\right) \epsilon_{2} \cdot k_{1} \epsilon_{3} \cdot k_{4}+\left(k_{3} \cdot k_{4}-\kappa_{3} \kappa_{4}\right) \epsilon_{2} \cdot k_{4} \epsilon_{3} \cdot k_{1}\right] \epsilon_{1} \cdot \epsilon_{4} \\
& +\left[\left(k_{2} \cdot k_{3}-\kappa_{2} \kappa_{3}\right) \epsilon_{2} \cdot k_{1} \epsilon_{4} \cdot k_{3}+\left(k_{3} \cdot k_{4}-\kappa_{3} \kappa_{4}\right) \epsilon_{2} \cdot k_{3} \epsilon_{4} \cdot k_{1}\right] \epsilon_{1} \cdot \epsilon_{3} \\
& \left.+\left[\left(k_{1} \cdot k_{3}-\kappa_{1} \kappa_{3}\right) \epsilon_{3} \cdot k_{2} \epsilon_{4} \cdot k_{1}+\left(k_{2} \cdot k_{3}-\kappa_{2} \kappa_{3}\right) \epsilon_{3} \cdot k_{1} \epsilon_{4} \cdot k_{2}\right] \epsilon_{1} \cdot \epsilon_{2}\right\} .
\end{aligned}
$$

As expected, this is simply the four-gluon amplitude with $k_{a} \cdot k_{b}$ replaced by $k_{a} \cdot k_{b}-\kappa_{a} \kappa_{b}$.

Mixed amplitudes containing both massive vector and adjoint scalar bosons in $d$ dimensions can also be obtained via dimensional reduction of massless gauge amplitudes in $d+M$ dimensions $[4,7]$. Massive adjoint scalar bosons arise from particles whose $(d+M)$ dimensional polarization and momentum vectors are given by ${ }^{3}$

$$
\mathcal{E}_{a}=\left(0 \mid 0, e_{a}\right), \quad K_{a}=\left(k_{a} \mid \kappa_{a}, 0\right) .
$$

\footnotetext{
${ }^{3}$ We choose the internal polarization of the would-be scalars to be orthogonal to the internal momenta of all the particles.
} 
Thus, invariants involving the adjoint scalars are given by

$$
K_{a} \cdot K_{b}=k_{a} \cdot k_{b}-\kappa_{a} \cdot \kappa_{b}, \quad \mathcal{E}_{a} \cdot K_{b}=0, \quad \mathcal{E}_{a} \cdot \mathcal{E}_{b}=e_{a} \cdot e_{b} .
$$

Again this implies that the amplitudes for massive vectors and scalars can be obtained from the corresponding massless amplitudes by simply replacing $k_{a} \cdot k_{b}$ with $k_{a} \cdot k_{b}-\kappa_{a} \cdot \kappa_{b}$.

The field theory corresponding to these dimensionally-reduced amplitudes contains adjoint scalar fields $\Phi_{i}{ }^{j}$, whose masses $g\left|v_{i}-v_{j}\right|$ arise from the $g^{2} \operatorname{Tr}\left([\Phi, H]^{2}\right)$ term in the Lagrangian [11]. As described above, the couplings in the theory are consistent with internal momentum conservation at each vertex. We have computed various mixed gaugefield and scalar four- and five-point amplitudes in the spontaneously-broken theory, and verified in all cases that the massive amplitudes are equivalent to the corresponding massless amplitudes with the replacement $k_{a} \cdot k_{b} \rightarrow k_{a} \cdot k_{b}-\kappa_{a} \kappa_{b}$, where $\kappa_{a}=g\left(v_{i_{a}}-v_{i_{a+1}}\right)$. This is not initially obvious from the Feynman diagram calculation in the spontaneously-broken theory, in which several different diagrams conspire to give the appropriate expressions, but is manifest from the dimensional reduction of the CHY representation of the amplitudes.

What if the internal momenta $\kappa_{a}$ of the particles span more than one dimension of the $M$-dimensional internal space? In this case, the dimensionally-reduced amplitudes appear to correspond to massive amplitudes in a spontaneously-broken gauge theory in which several adjoint Higgs fields $H^{I}, I=1, \cdots, h$ have nonzero vacuum expectation values $\left\langle H^{I}\right\rangle=\operatorname{diag}\left(v_{1}^{I}, v_{2}^{I}, \cdots, v_{N}^{I}\right)$ that are mutually commuting. In this theory, the off-diagonal gauge bosons $W_{i}^{j}$ obtain masses $g\left[\sum_{I=1}^{h}\left(v_{i}^{I}-v_{j}^{I}\right)^{2}\right]^{1 / 2}$. We identify the internal momentum $\kappa_{i j}^{I}$ of $W_{i}^{j}$ with $g\left(v_{i}^{I}-v_{j}^{I}\right)$, automatically satisfying the triple vertex constraint $\kappa_{i j}^{I}+\kappa_{j k}^{I}=\kappa_{i k}^{I}$. Although momentum conservation still requires $\sum_{a=1}^{n} \kappa_{a}^{I}=0$, the constraint on the masses of the external particles is considerably weakened compared to the case in which only one Higgs field gets a vev.

Finally, we recall that momentum conservation can be used to express all kinematic invariants $k_{a} \cdot k_{b}$ of an $n$-point amplitude in terms of an independent set of $n(n-3) / 2$ invariants

$$
k_{1} \cdot k_{c}, \quad k_{2} \cdot k_{c}, \quad k_{c} \cdot k_{d}, \quad c, d \in\{3, \cdots, n-1\} .
$$

If we consider $n$-point amplitudes with at most three massive particles $(a=1,2$, and $n$ ), then $\kappa_{c}=0$ for $c \in\{3, \cdots, n-1\}$ and all of the invariants in eq. (12) are unchanged under $k_{a} \cdot k_{b} \rightarrow k_{a} \cdot k_{b}-\kappa_{a} \cdot \kappa_{b}$. Consequently, the expressions for amplitudes with at most three massive particles, when expressed in terms of $\epsilon_{a} \cdot k_{b}, \epsilon_{a} \cdot \epsilon_{b}$, and the invariants (12), are identical to the corresponding massless amplitudes, as found in ref. [7]. For example, eqs. (8) and (9) reduce to the massless amplitude when $m_{3}$, and therefore $\kappa_{3}$, vanishes.

In this note, we have argued that dimensionally-reduced tree-level gauge-theory amplitudes of massless particles possessing internal momentum $\kappa$ are equivalent to tree-level amplitudes of massive particles in a lower-dimensional gauge theory in which the gauge symmetry is spontaneously broken by an adjoint Higgs field. The same procedure can be applied to obtain amplitudes of massive particles in other theories with a CHY representation, including gravity, Dirac-Born-Infeld theory, and the nonlinear $\sigma$ model. It will be intriguing to determine the lower-dimensional theories to which these massive amplitudes belong. 


\section{Acknowledgments}

The author wishes to thank Freddy Cachazo, Song He, and Ellis Yuan for fruitful discussions. This material is based upon work supported in part by the National Science Foundation under Grant No. PHY14-16123.

Open Access. This article is distributed under the terms of the Creative Commons Attribution License (CC-BY 4.0), which permits any use, distribution and reproduction in any medium, provided the original author(s) and source are credited.

\section{References}

[1] F. Cachazo, S. He and E.Y. Yuan, Scattering of massless particles in arbitrary dimensions, Phys. Rev. Lett. 113 (2014) 171601 [arXiv:1307.2199] [INSPIRE].

[2] F. Cachazo, S. He and E.Y. Yuan, Scattering of massless particles: scalars, gluons and gravitons, JHEP 07 (2014) 033 [arXiv:1309.0885] [INSPIRE].

[3] F. Cachazo, S. He and E.Y. Yuan, Einstein-Yang-Mills scattering amplitudes from scattering equations, JHEP 01 (2015) 121 [arXiv: 1409.8256] [INSPIRE].

[4] F. Cachazo, S. He and E.Y. Yuan, Scattering equations and matrices: from Einstein to Yang-Mills, DBI and NLSM, JHEP 07 (2015) 149 [arXiv:1412.3479] [INSPIRE].

[5] C. Kalousios, Scattering equations, generating functions and all massless five point tree amplitudes, JHEP 05 (2015) 054 [arXiv: 1502.07711] [INSPIRE].

[6] F. Cachazo and H. Gomez, Computation of contour integrals on $\mathcal{M}_{0, n}$, arXiv:1505.03571 [INSPIRE].

[7] S.G. Naculich, CHY representations for gauge theory and gravity amplitudes with up to three massive particles, JHEP 05 (2015) 050 [arXiv:1501.03500] [INSPIRE].

[8] L. Dolan and P. Goddard, Proof of the formula of Cachazo, He and Yuan for Yang-Mills tree amplitudes in arbitrary dimension, JHEP 05 (2014) 010 [arXiv:1311.5200] [INSPIRE].

[9] L. Dolan and P. Goddard, The polynomial form of the scattering equations, JHEP 07 (2014) 029 [arXiv: 1402.7374] [INSPIRE].

[10] S.G. Naculich, Scattering equations and BCJ relations for gauge and gravitational amplitudes with massive scalar particles, JHEP 09 (2014) 029 [arXiv: 1407.7836] [INSPIRE].

[11] L.F. Alday, J.M. Henn, J. Plefka and T. Schuster, Scattering into the fifth dimension of $N=4$ super Yang-Mills, JHEP 01 (2010) 077 [arXiv:0908.0684] [INSPIRE].

[12] J.M. Henn, S.G. Naculich, H.J. Schnitzer and M. Spradlin, Higgs-regularized three-loop four-gluon amplitude in N=4 SYM: exponentiation and Regge limits, JHEP 04 (2010) 038 [arXiv: 1001.1358] [INSPIRE].

[13] J.M. Henn, S.G. Naculich, H.J. Schnitzer and M. Spradlin, More loops and legs in Higgs-regulated $N=4$ SYM amplitudes, JHEP 08 (2010) 002 [arXiv:1004.5381] [INSPIRE].

[14] K.G. Selivanov, An Infinite set of tree amplitudes in Higgs-Yang-Mills, Phys. Lett. B 460 (1999) 116 [hep-th/9906001] [INSPIRE].

[15] R.H. Boels, No triangles on the moduli space of maximally supersymmetric gauge theory, JHEP 05 (2010) 046 [arXiv: 1003.2989] [INSPIRE]. 
[16] Z. Bern, J.J. Carrasco, T. Dennen, Y.-t. Huang and H. Ita, Generalized unitarity and six-dimensional helicity, Phys. Rev. D 83 (2011) 085022 [arXiv:1010.0494] [InSPIRE].

[17] N.E.J. Bjerrum-Bohr, J.F. Donoghue and P. Vanhove, On-shell techniques and universal results in quantum gravity, JHEP 02 (2014) 111 [arXiv:1309.0804] [INSPIRE].

[18] J. Plefka, T. Schuster and V. Verschinin, From six to four and more: massless and massive maximal super Yang-Mills amplitudes in $6 D$ and $4 D$ and their hidden symmetries, JHEP 01 (2015) 098 [arXiv: 1405.7248] [INSPIRE].

[19] L.F. Alday and J.M. Maldacena, Gluon scattering amplitudes at strong coupling, JHEP 06 (2007) 064 [arXiv:0705.0303] [INSPIRE].

[20] H. Kawai and T. Suyama, Some implications of perturbative approach to AdS/CFT correspondence, Nucl. Phys. B 794 (2008) 1 [arXiv:0708.2463] [INSPIRE].

[21] R.M. Schabinger, Scattering on the moduli space of $N=4$ super Yang-Mills, arXiv:0801.1542 [INSPIRE].

[22] J. McGreevy and A. Sever, Planar scattering amplitudes from Wilson loops, JHEP 08 (2008) 078 [arXiv:0806.0668] [INSPIRE].

[23] N. Berkovits and J. Maldacena, Fermionic T-duality, dual superconformal symmetry and the amplitude/Wilson loop connection, JHEP 09 (2008) 062 [arXiv:0807.3196] [INSPIRE].

[24] N. Craig, H. Elvang, M. Kiermaier and T. Slatyer, Massive amplitudes on the Coulomb branch of $N=4$ SYM, JHEP 12 (2011) 097 [arXiv:1104.2050] [INSPIRE].

[25] M. Kiermaier, The Coulomb-branch S-matrix from massless amplitudes, arXiv:1105.5385 [INSPIRE]. 